\title{
Baseline participant characteristics and risk for dropout from 10 obesity randomized controlled trials: a pooled analysis of individual level data
}

\author{
Kathryn Ann Kaiser ${ }^{1,2}$ *, Olivia Affuso ${ }^{2,3}$, Renee Desmond ${ }^{2,4}$ and David B. Allison ${ }^{1,2}$ * \\ 1 School of Public Health, University of Alabama at Birmingham, Birmingham, AL, USA \\ ${ }^{2}$ Nutrition Obesity Research Center, University of Alabama at Birmingham, Birmingham, AL, USA \\ ${ }^{3}$ Department of Epidemiology, School of Public Health, University of Alabama at Birmingham, Birmingham, AL, USA \\ ${ }^{4}$ Department of Preventive Medicine, School of Medicine, University of Alabama at Birmingham, Birmingham, AL, USA
}

Edited by:

Johannes Le Coutre, Nestlé Research

Center, Switzerland

\section{Reviewed by:}

Erin Hennessy, Leidos Biomedical

Research, Inc., USA

Amanda Willig, University of Alabama

at Birmingham, USA

\section{*Correspondence:}

Kathryn Ann Kaiser and David B. Allison, School of Public Health, University of Alabama at Birmingham, Ryals Public Health Building, Room 140J, 1665 University Boulevard, Birmingham, AL 35294, USA

e-mail:kakaiser@uab.edu
Introduction: Understanding participant demographic characteristics that inform the optimal design of obesity randomized controlled trials (RCTs) have been examined in few studies. The objective of this study was to investigate the association of individual participant characteristics and dropout rates (DORs) in obesity RCTs by pooling data from several publicly available datasets for analyses. We comprehensively characterize DORs and patterns in obesity RCTs at the individual study level, and describe how such rates and patterns vary as a function of individual level characteristics.

Methods: We obtained and analyzed nine publicly available, obesity RCT datasets that examined weight loss or weight gain prevention as a primary or secondary endpoint. Four risk factors for dropout were examined by Cox proportional hazards including sex, age, baseline BMI, and race/ethnicity. The individual study data were pooled in the final analyses with a random effect for study, and $\mathrm{HR}$ and $95 \% \mathrm{Cls}$ were computed.

Results: Results of the multivariate analysis indicated that the risk of dropout was significantly higher for females compared to males $(H R=1.24,95 \% \mathrm{Cl}=1.05,1.46)$. Hispanics and Non-Hispanic blacks had a significantly higher dropout rate compared to non-Hispanic whites ( $\mathrm{HR}=1.62,95 \% \mathrm{Cl}=1.37,1.91 ; \mathrm{HR}=1.22,95 \% \mathrm{Cl}=1.11,1.35$, respectively). There was a significantly increased risk of dropout associated with advancing age $(\mathrm{HR}=1.02$, $95 \% \mathrm{Cl}=1.01,1.02)$ and increasing $\mathrm{BMI}(\mathrm{HR}=1.03,95 \% \mathrm{Cl}=1.03,1.04)$.

\begin{abstract}
Conclusion/Significance: As more studies may focus on special populations, researchers designing obesity RCTs may wish to oversample in certain demographic groups if attempting to match comparison groups based on generalized estimates of expected DORs, or otherwise adjust a priori power estimates. Understanding true reasons for dropout may require additional methods of data gathering not generally employed in obesity RCTs, e.g., time on treatment.
\end{abstract}

Keywords: obesity, pooled analysis, randomized trials, dropout, participant characteristics

\section{INTRODUCTION}

Dropout is a major problem in studies of weight loss interventions (1). Identification of predictors of dropout could be important to enhance recruitment in vulnerable groups, as well as to develop strategies to prevent dropout among those at high risk. Previous investigations in single studies have reported baseline factors that are associated with dropout including sex, age, marital status and race, e.g., Ref. (2), or the presence of baseline comorbidities such as Type 2 diabetes (3). Psychological predictors of dropout such as motivation and stages of change have also been investigated as factors, with little evidence of reliable predictive value across multiple studies for many of the variables proposed (4). The purpose of this investigation is to conduct a pooled meta-analysis to identify baseline factors that are related to study retention among a large cohort of subjects with racial/ethnic, age, sex, and body weight heterogeneity.

\section{MATERIALS AND METHODS STUDY SAMPLES}

For this investigation, individual level participant data from the selected studies were obtained from the Biologic Specimen and Data Repository Information Coordinating Center (BioLINCC), for the National Heart, Lung, and Blood Institute (5) and from the National Institute for Digestive and Diseases of the Kidney (NIDDK) Central Data Repository (https://www.niddkrepository. org). Searches were performed for studies meeting the inclusion criteria defined as: the interventions were dietary and/or physical activity interventions in free living people of any age, an outcome 
of interest was body weight, and basic demographic information such as age, gender, and race were available in most records. Ten were selected for inclusion (6-16). The investigation was approved for secondary data analysis by the Institutional Review Board at the University of Alabama at Birmingham.

\section{DATA DEFINITIONS AND RECODING}

The raw, de-identified datasets obtained were standardized for consistency in coding prior to pooling. Variable codes were assigned as follows: sex $(0=$ male, $1=$ female $)$; race $(0=$ White, Non-minority or non-Black, $1=$ Black, $2=$ Asian, $3=$ Hispanic, $4=$ other); age (continuous coded in years); body mass index (BMI - continuous); dropout $(0=$ No, $1=$ Yes for any time before the protocol was completed), follow-up time (months). Treatment groups were coded as nominal variables within each study. The individual study frequency data were examined and compared to study publications for accuracy (6-16).

\section{STATISTICAL ANALYSIS}

Descriptive statistics including means and standard deviations (SD) for continuous data and frequency counts for categorical predictors were generated by study as well as for the overall analyses. The Cox model was used to estimate hazard ratios (HR) for risk of dropout within each study. Proportionality assumptions were assessed within each study by including a predictor term in the model for the interaction of the predictors with time. If a time-dependent covariate was significant, this could indicate a violation of the proportionality assumption for that specific predictor. A Martingale residual analysis was used to examine whether the functional form of the linear predictors was appropriate or whether a quadratic term would improve model fit $(17,18)$.

For the combined analysis, the participant-level raw data were pooled from the multiple studies. Two types of pooled analyses were performed. The preliminary analyses combined the studyspecific HR and standard errors using the SAS METAANAL macro (18) to produce the DerSimonian-Laird (19) estimator for random effects. Plots of the study specific estimates as well as the overall random effects model were visually inspected for heterogeneity among the studies for estimates of BMI, age, sex, and race/ethnicity. Due to the significant heterogeneity detected on the random effects models for univariate predictors, the final pooled model using a combined dataset was run with PHREG $^{\circledR}$ (SAS Ver. 9.2, Cary, NC, USA) using study as a random effect. Further multivariate Cox models were analyzed to include categorical terms for BMI $(<35,35-39.9, \geq 40)$ and age $(<25,25-64.9, \geq 65)$ while controlling for sex and race/ethnicity.

\section{RESULTS}

Table 1 summarizes the studies included in the meta-analysis including a brief description, the endpoint for determining censoring for dropout and the dropout proportion. The descriptive characteristics for the covariates included in this investigation are shown in Table 2. The results of the pooled analyses are in Table 3 (using age and BMI as continuous variables) and in Table 4 (using age and BMI as categorical variables).

The results of univariate models including single factors in the model for each study are shown in Tables 5-8. The testing of residuals for age and BMI in each study did not show a significant departure from expected simulations. In the Dietary Intervention Study in Children (DISC) study, there was some indication of a poor fit for a linear model for age, although this study involved children aged $8-10$ years old. The proportionality assumptions for the interaction terms of gender by time, race by time, BMI by time, and age by time were tested within each study. The results showed a statistically significant interaction for gender by time in the Dietary Approaches to Stop Hypertension (DASH) study and TOHP1 study, and race by time in the Diabetes Prevention Program (DPP) study. No other interaction terms were significant.

\section{POOLED ANALYSES}

The combined cohort for pooled analyses consisted of 75,764 subjects and the overall dropout percentage was $5.2 \%(n=3821)$. The distribution by gender was 53,938 females $(71.2 \%)$ and 21,826 males $(28.8 \%)$. The majority were non-Hispanic white $(81.9 \%)$, followed by non-Hispanic black (11.4\%), Asian (3.2\%), and Hispanic (1.9\%). The mean age was 56.4 years (SD 11.1) and mean baseline BMI was $28.9(\mathrm{SD}=5.45)$.

Results of the multivariate analysis (Table 3 ) indicate that the risk of dropout was significantly higher for females compared to males $(\mathrm{HR}=1.24,95 \%$ CI $1.0,1.46)$. Non-Hispanic blacks had a significantly higher dropout rate compared to nonHispanic whites $(\mathrm{HR}=1.22,95 \%$ CI 1.11, 1.35) and Hispanics were also significantly higher compared to non-Hispanic whites $(\mathrm{HR}=1.62,95 \% \mathrm{CI} 1.37,1.91)$. There was a statistically significant increased risk of dropout associated with advancing age (Hazard Ratio $=1.01)$ and increasing BMI $(\mathrm{HR}=1.03)$. The Wald test for the random effect of study was significant $(p<0.001)$. Using age as a categorical variable showed an increased risk of dropout for subjects aged 65 years and over compared to those aged 25-64 years $(\mathrm{HR}=1.37 ; 95 \% \mathrm{CI}=1.26-1.49)$. Also individuals who were categorized as obese class II and obese class III (20) were more likely to dropout compared to those who were categorized as overweight or obese class I ( $\mathrm{HR}=1.40$ and 1.69, respectively; Table 4). Figure 1 below shows the combined, overall dropout survival probability using Cox proportional hazards projections. Figures 2-5 show individual study hazard ratios of risk for drop out using BMI, age, gender or race as predictors, respectively.

\section{SENSITIVITY ANALYSIS}

Because there were some inter-study definitions that were not consistent for race [e.g., the DASH study coded race only as minority vs. non-minority and the Lipids Research Clinics (LRC) and PREMIER studies coded race as Black vs. Non-Black], a sensitivity analysis was conducted excluding these studies. An analysis excluding the LRC and PREMIER studies did not show any appreciable difference in the results from the random effects Cox model. A second analysis excluding only the DASH study did not show any significant differences from the combined model (data not shown). Because our analysis included one study of children, who may have differing factors that determine study retention, we performed an additional sensitivity analysis excluding the DISC study. There were no meaningful differences in the results or interpretation following exclusion of the DISC data. 
Table 1 | Endpoint determination and reference used for included studies.

\begin{tabular}{|c|c|c|c|c|}
\hline Study title & Study description & Dropout determination & $\begin{array}{l}\text { Dropout } \\
n(\%)\end{array}$ & $\begin{array}{l}\text { Published } \\
\text { missingness } n(\%)\end{array}$ \\
\hline $\begin{array}{l}\text { Activity Counseling } \\
\text { Trial (ACT) (11) }\end{array}$ & $\begin{array}{l}\text { The ACT was a multicenter, randomized, controlled } \\
\text { trial to evaluate the effects of two primary care } \\
\text { based activity counseling regimens on physical } \\
\text { activity and cardiorespiratory fitness after } \\
24 \text { months when compared with a standard. }\end{array}$ & $\begin{array}{l}\text { Subjects who did not complete } \\
\text { assessment at } 2 \text { years }\end{array}$ & $81(9.3)$ & $\begin{array}{l}75(8.6) \text { had no } \\
\text { assessment at } \\
24 \text { months }\end{array}$ \\
\hline $\begin{array}{l}\text { Dietary Approaches } \\
\text { to stop hypertension } \\
\text { (DASH) (10) }\end{array}$ & $\begin{array}{l}\text { The DASH trial was a multicenter, randomized } \\
\text { feeding study that tested the effects of dietary } \\
\text { patterns on blood pressure. The intervention phase } \\
\text { was an 8-week period in which the subjects } \\
\text { followed their assigned diets. }\end{array}$ & $\begin{array}{l}\text { Subjects who did not complete the } \\
\text { assessment at } 8 \text { weeks }\end{array}$ & $17(3.7)$ & $\begin{array}{l}13(2.8) \text { percentage } \\
\text { who did not } \\
\text { complete } \\
\text { intervention phase }\end{array}$ \\
\hline $\begin{array}{l}\text { Dietary Intervention } \\
\text { Study in Children } \\
\text { (DISC) (7) }\end{array}$ & $\begin{array}{l}\text { The DISC study was a controlled clinical trial to } \\
\text { examine the efficacy and safety of long-term } \\
\text { dietary intervention for reduction of LDL-C in } \\
\text { pubescent children. }\end{array}$ & $\begin{array}{l}\text { Subjects who did not attend the 3-year } \\
\text { lipid assessment visit }\end{array}$ & $43(6.5)$ & $\begin{array}{l}39 \text { (5.9) number } \\
\text { with no outcome } \\
\text { data at } 3 \text { years }\end{array}$ \\
\hline $\begin{array}{l}\text { Diabetes Prevention } \\
\text { Program (DPP) (13) }\end{array}$ & $\begin{array}{l}\text { A randomized trial was conducted to compare a } \\
\text { lifestyle intervention or metformin on the } \\
\text { development of diabetes. }\end{array}$ & $\begin{array}{l}\text { The blinded treatment was terminated } \\
\text { early so dropout was assessed by an } \\
\text { algorithm provided by the investigator } \\
\text { (personal communication to author } \\
\text { Renee Desmond) }\end{array}$ & $149(4.1)$ & $\begin{array}{l}246(7.6) \\
\text { percentage who } \\
\text { had no follow-up } \\
\text { within last } \\
5 \text { months }\end{array}$ \\
\hline $\begin{array}{l}\text { Lipids Research } \\
\text { Clinics (LRC) } \\
\text { Coronary Primary } \\
\text { Prevention Trial (14) }\end{array}$ & $\begin{array}{l}\text { The LRC CPPT was a multicenter, randomized, } \\
\text { double-blind clinical trial on the efficacy of } \\
\text { cholesterol lowering. }\end{array}$ & $\begin{array}{l}\text { Subjects who did not complete the } \\
\text { minimum follow-up of } 7 \text { years }\end{array}$ & $214(5.7)$ & Not reported \\
\hline $\begin{array}{l}\text { Multiple Risk Factor } \\
\text { Intervention Trial } \\
\text { (MRFIT) (12) }\end{array}$ & $\begin{array}{l}\text { MRFIT was a randomized, primary prevention trial } \\
\text { to test whether lowering elevated serum } \\
\text { cholesterol and diastolic blood pressure and } \\
\text { ceasing cigarette smoking would reduce coronary } \\
\text { heart disease mortality. }\end{array}$ & $\begin{array}{l}\text { Subjects who did not complete a } \\
\text { follow-up period of at least } 6 \text { years }\end{array}$ & $1003(7.8)$ & Not reported \\
\hline PREMIER (9) & $\begin{array}{l}\text { The objective of the randomized trial was to } \\
\text { determine the effect on blood pressure to two } \\
\text { multi-component behavioral interventions. }\end{array}$ & $\begin{array}{l}\text { Subjects who did not complete the } \\
\text { primary outcome assessment at } \\
6 \text { months and did not complete the } \\
\text { 18-month intervention }\end{array}$ & $139(17.2)$ & $\begin{array}{l}45 \text { (5.5) did not } \\
\text { complete } 6 \text { months } \\
\text { follow-up although } \\
\text { intervention lasted } \\
18 \text { months }\end{array}$ \\
\hline $\begin{array}{l}\text { Trials of Hypertension } \\
\text { (TOHPI Lifestyle and } \\
\text { II) (6) }\end{array}$ & $\begin{array}{l}\text { Three lifestyle change groups were compared with } \\
\text { unmasked non-intervention controls over } \\
18 \text { months to assess change in diastolic blood } \\
\text { pressure. Phase II TOHP was a multicenter } \\
\text { randomized controlled clinical trial with a } 2 \times 2 \\
\text { factorial design. }\end{array}$ & $\begin{array}{l}\text { Subjects who did not complete an } \\
\text { 18-month follow-up visit (Lifestyle } \\
\text { TOHP I) or subjects who did not } \\
\text { complete a 36-month follow-up visit } \\
\text { (TOPH II) }\end{array}$ & $\begin{array}{l}92(6.3) \\
243(10.2)\end{array}$ & $\begin{array}{l}37 \text { (4.2) of active } \\
\text { arms did not } \\
\text { complete follow-up } \\
273 \text { (11.5) did not } \\
\text { have weight at } \\
36 \text { months }\end{array}$ \\
\hline $\begin{array}{l}\text { Women's Health } \\
\text { Initiative (WHI) } \\
\text { Dietary Modification } \\
\text { Trial (15) }\end{array}$ & $\begin{array}{l}\text { The WHI dietary modification trial was designed to } \\
\text { examine the benefits and risk of a dietary pattern } \\
\text { low in fat on various outcomes in postmenopausal } \\
\text { women. }\end{array}$ & $\begin{array}{l}\text { Subjects who were deceased or who } \\
\text { stopped follow-up before } 7 \text { years }\end{array}$ & $2242(4.6)$ & $\begin{array}{l}4071 \text { (8.3) } \\
\text { withdrew, lost, or } \\
\text { deceased }\end{array}$ \\
\hline
\end{tabular}

\section{DISCUSSION}

Meta-analysis of individual level data has an advantage; in that, a research question can be addressed that was not part of the original research investigation. By obtaining the individual data, common definitions can be used for coding variables and adjustments for confounders may be performed. Obviously, the power for the meta-analysis is greater than for the individual studies from which the data are compiled. As such, the present analysis may be viewed 
Table 2 | Characteristics of included studies

\begin{tabular}{|c|c|c|c|c|c|c|c|c|c|c|}
\hline Factor & ACT & DASH & DISC & DPP & LRC & MRFIT & PREMIER & TOHP I & TOHP II & WHI \\
\hline \multicolumn{11}{|l|}{ Gender } \\
\hline Male & $479(54.8)$ & $234(51.0)$ & $362(54.6)$ & $362(54.6)$ & $3,774(100.0)$ & $12,866(100.0)$ & $310(38.3)$ & $1,037(70.7)$ & $1,566(65.7)$ & $0(0.0)$ \\
\hline Female & $395(45.2)$ & $225(49.0)$ & $301(45.4)$ & $301(45.4)$ & $0(0.0)$ & $0(0.0)$ & $500(61.7)$ & $429(29.3)$ & 816 (34.3) & $48,835(100.0)$ \\
\hline \multicolumn{11}{|l|}{ Race/ethnicity } \\
\hline Non-Hispanic White & 597 (68.9) & $156(34.0)$ & $574(86.6)$ & $2,117(57.8)$ & $3,601(96.6)$ & 11,559 (89.8) & $531(65.6)$ & $1,182(80.6)$ & 1,888 (79.3) & 39,762 (81.6) \\
\hline Non-Hispanic Black & $217(25.0)$ & $303(66.0)$ & $56(8.5)$ & $751(20.5)$ & $125(3.4)$ & 931 (7.3) & $279(34.4)$ & $252(17.2)$ & $421(17.7)$ & $5,262(10.8)$ \\
\hline Asian & $34(3.9)$ & $0(0.0)$ & $33(5.0)$ & $0(0.0)$ & $0(0.0)$ & $0(0.0)$ & $0(0.0)$ & $0(0.0)$ & $73(3.1)$ & $1,105(2.3)$ \\
\hline Hispanic & $0(0.0)$ & $0(0.0)$ & $0(0.0)$ & 609 (16.6) & $0(0.0)$ & $0(0.0)$ & $0(0.0)$ & $0(0.0)$ & $0(0.0)$ & 1,845 (3.8) \\
\hline Other & $19(2.2)$ & $0(0.0)$ & $0(0.0)$ & $188(5.1)$ & $0(0.0)$ & $376(2.9)$ & $0(0.0)$ & $32(2.2)$ & $0(0.0)$ & $762(1.6)$ \\
\hline \multicolumn{11}{|l|}{$\mathrm{BMI}$} \\
\hline$<18$ & $4(0.5)$ & $0(0.0)$ & $459(69.2)$ & $0(0.0)$ & $6(0.2)$ & $21(0.1)$ & $0(0.0)$ & $5(0.3)$ & $0(0.0)$ & $86(0.2)$ \\
\hline $18.5-24.9$ & $210(24.3)$ & $114(24.8)$ & $202(30.5)$ & $0(0.0)$ & 1,215 & $2,649(20.6)$ & $39(4.8)$ & $354(24.2)$ & $24(1.0)$ & $12,551(25.8)$ \\
\hline $25.0-29.9$ & $303(35.1)$ & $179(39.0)$ & $2(0.3)$ & $1,199(32.7)$ & $2,215(59.2)$ & 7,125 (55.4) & 242 (29.9) & 694 (47.3) & $1,014(42.6)$ & 17,425 (35.8) \\
\hline$\geq 30$ & $346(40.1)$ & $166(36.2)$ & $0(0.0)$ & 2,466 (67.3) & 305 (8.1) & $3,071(23.9)$ & 529 (65.3) & $413(28.2)$ & $1,344(56.4)$ & $18,591(38.2)$ \\
\hline${ }^{*}$ Obese class I & $208(59.2)$ & 158 (95.2) & $0(0.0)$ & 915 (37.1) & $305(100.0)$ & 2659 (86.6) & 241 (45.5) & $372(90.1)$ & 1,048 (78.0) & $11,216(60.3)$ \\
\hline${ }^{*}$ Obese class II & 89 (23.6) & $8(4.8)$ & $0(0.0)$ & $940(38.1)$ & $0(0.0)$ & $404(13.2)$ & 469 (32.0) & 41 (9.9) & 296 (22.0) & $5,046(27.1)$ \\
\hline${ }^{*}$ Obese class III & 49 (14.5) & $0(0.0)$ & $0(0.0)$ & $611(24.8)$ & $0(0.0)$ & $8(0.2)$ & 119 (22.5) & $0(0.0)$ & $0(0.0)$ & 2,329 (12.5) \\
\hline \multicolumn{11}{|l|}{ Age } \\
\hline$<29$ & $0(0.0)$ & 49 (10.7) & $663(100.0)$ & $0(0.0)$ & $0(0.0)$ & $0(0.0)$ & $11(1.4)$ & $0(0.0)$ & $0(0.0)$ & $0(0.0)$ \\
\hline $30-49$ & $434(49.7)$ & 283 (61.6) & $0(0.0)$ & $1,811(49.4)$ & $2,257(60.3)$ & $8,670(67.4)$ & $426(52.6)$ & $1,162(79.3)$ & 1,899 (79.7) & $2(0.0)$ \\
\hline $50-69$ & $403(46.1)$ & $118(25.7)$ & $0(0.0)$ & $1,854(50.6)$ & $1,487(39.7)$ & 4,196 (32.6) & 359 (44.3) & $304(20.7)$ & 483 (20.3) & 40,711 (83.4) \\
\hline$>70$ & $37(4.2)$ & $9(2.0)$ & $0(0.0)$ & $0(0.0)$ & $0(0.0)$ & $0(0.0)$ & $14(1.7)$ & $0(0.0)$ & $0(0.0)$ & $8,122(16.6)$ \\
\hline BMI (mean, SD) & $29.4(6.0)$ & $28.2(3.8)$ & $17.5(2.4)$ & 33.5 (95.7) & $26.2(2.6)$ & 27.7 (3.5) & $33.1(5.7)$ & $27.8(3.7)$ & $30.9(3.1)$ & $29.1(5.7)$ \\
\hline Age (mean, SD) & $50.9(10.0)$ & $44.6(10.7)$ & $9.6(0.7)$ & $50.5(9.3)$ & $47.2(6.5)$ & $46.2(6.0)$ & $50.0(9.1)$ & $43.0(6.5)$ & $43.6(6.2)$ & $62.3(6.9)$ \\
\hline
\end{tabular}

${ }^{*} n(\%)$ within obese

ACT, Activity Counseling Trial (11); DASH, Dietary Approaches to Stop Hypertension (10); DISC, Dietary Intervention Study in Children (7); DPP, Diabetes Prevention Program (13); LRC, Lipids Research Clinics Coronary Primary Prevention Trial (14); MRFIT, Multiple Risk Factor Intervention Trial (12); PREMIER (9); TOHPI, Trials of Hypertension (Lifestyle I and II) (6) WHI, Women's Health Initiative Dietary Modification Trial (15).

All values $n(\%)$ 
Table 3 | Results of pooled analysis of individual data for dropout risk by baseline characteristics $(n=75,764)$.

\begin{tabular}{lcccc}
\hline Factor & $\begin{array}{l}\text { Parameter } \\
\text { estimate }\end{array}$ & $\begin{array}{l}\text { Standard } \\
\text { error }\end{array}$ & $\boldsymbol{p}$ & HR (95\% Cl) \\
\hline $\begin{array}{l}\text { Gender } \\
\text { Male }\end{array}$ & - & & & \\
$\quad$ Female & 0.21435 & 0.08517 & 0.0118 & $1.24(1.05,1.46)$ \\
Race & & & & \\
$\quad$ Non-Hispanic White & - & - & - & 1 \\
Non-Hispanic Black & 0.20127 & 0.04888 & $<0.0001$ & $1.22(1.11,1.35)$ \\
Asian & 0.21775 & 0.13263 & 0.1006 & $1.24(0.96,1.61)$ \\
Hispanic & 0.48028 & 0.08424 & $<0.0001$ & $1.62(1.37,1.91)$ \\
$\quad$ Other & 0.24451 & 0.11038 & 0.0268 & $1.28(1.03,1.56)$ \\
Age (years) & 0.01446 & 0.00237 & $<0.0001$ & $1.02(1.01,1.02)$ \\
BMl (units) & 0.03097 & 0.00303 & $<0.0001$ & $1.03(1.03,1.04)$ \\
\hline
\end{tabular}

Table 4 | Results of pooled analysis of individual data for dropout risk by baseline characteristics using categorical variables for age and BMI $(n=75,764)$

\begin{tabular}{lcccc}
\hline Factor & $\begin{array}{l}\text { Parameter } \\
\text { estimate }\end{array}$ & $\begin{array}{l}\text { Standard } \\
\text { error }\end{array}$ & $\boldsymbol{P}$ & HR (95\% Cl) \\
\hline $\begin{array}{l}\text { Gender } \\
\text { Male }\end{array}$ & & & & \\
$\quad$ Female & - & & & 1 \\
Race & 0.19961 & 0.08539 & 0.0194 & $1.22(1.03,1.44)$ \\
Non-Hispanic White & - & - & - & 1 \\
Non-Hispanic Black & 0.22306 & 0.04874 & $<0.0001$ & $1.25(1.14,1.38)$ \\
Asian & 0.16679 & 0.13237 & 0.2077 & $1.18(0.91,1.53)$ \\
Hispanic & 0.50321 & 0.08426 & $<0.0001$ & $1.65(1.40,1.95)$ \\
Other & 0.24367 & 0.11039 & 0.0273 & $1.28(1.03,1.58)$ \\
Age (years) & & & & \\
$<25$ years & 1.19314 & 0.6954 & 0.0862 & $3.30(0.84,12.86)$ \\
$25-64$ years & - & - & - & - \\
$\geq 65$ years & 0.31667 & 0.04184 & $<0.0001$ & $1.37(1.26,1.49)$ \\
BMI (units) & & & & \\
$<35$ Class I or less & - & - & - & - \\
35-40 Class II & 0.33444 & 0.05248 & $<0.0001$ & $1.39(1.26,1.55)$ \\
$>40$ Class III & 0.52731 & 0.07047 & $<0.0001$ & $1.69(1.48,1.95)$ \\
\hline
\end{tabular}

as presenting very good estimates of the demographic and baseline characteristics that may be associated with increased dropout rates (DORs) in weight loss randomized controlled trials (RCTs).

Some disadvantages of performing a meta-analysis of this type are that the results may not reflect all populations and the metaanalysis itself may be subject to bias because some studies may be excluded due to unavailability of raw data. If internal errors or inconsistency are detected in the analysis, these issues may not be able to be resolved, especially if the data are for public use and tracing back to individual records is not possible. An examination of the survival in study of a small single trial $(N=91$ at baseline) described the distribution of attrition to be exponential, with
Table 5 | Summary of effect estimates for Body Mass Index (BMI) by study and estimates of effects in meta-analyses of dropout.

\begin{tabular}{lcccc}
\hline Study & $\begin{array}{l}\text { Parameter } \\
\text { estimate }\end{array}$ & $\begin{array}{l}\text { Standard } \\
\text { error }\end{array}$ & $\begin{array}{l}\text { Weight in fixed } \\
\text { effects model }\end{array}$ & $\begin{array}{l}\text { Weight in random } \\
\text { effects model }\end{array}$ \\
\hline ACT & 0.07021 & 0.01676 & 0.02754 & 0.11135 \\
LRC & -0.01250 & 0.02669 & 0.01086 & 0.05682 \\
DASH & -0.04058 & 0.06338 & 0.00193 & 0.01196 \\
DISC & 0.16774 & 0.05975 & 0.00217 & 0.01339 \\
DPP & -0.01343 & 0.00467 & 0.03471 & 0.12785 \\
MRFIT & 0.02471 & 0.01028 & 0.07321 & 0.18225 \\
PREMIER & 0.02035 & 0.01529 & 0.03309 & 0.12439 \\
TOHPI & 0.01138 & 0.03193 & 0.00759 & 0.04213 \\
TOHP II & 0.00347 & 0.02925 & 0.00904 & 0.04887 \\
WHI & 0.02966 & 0.00311 & 0.79987 & 0.28076 \\
\hline
\end{tabular}

Summary parameter estimate 0.0289 (SE $=0.0071) p=<0.00001$.

$Q$ statistic for heterogeneity with 9 degrees of freedom $=16.66, p=0.0543$.

Table 6 | Summary of effect estimates for age by study and estimates of effects in meta-analyses of dropout.

\begin{tabular}{lrrcc}
\hline Study & $\begin{array}{l}\text { Parameter } \\
\text { estimate }\end{array}$ & \multicolumn{1}{l}{$\begin{array}{l}\text { Standard } \\
\text { error }\end{array}$} & $\begin{array}{l}\text { Weight in fixed } \\
\text { effects model }\end{array}$ & $\begin{array}{l}\text { Weight in random } \\
\text { effects model }\end{array}$ \\
\hline ACT & -0.01220 & 0.01223 & 0.03788 & 0.11078 \\
LRC & 0.01437 & 0.01063 & 0.05015 & 0.11780 \\
DASH & -0.01362 & 0.02348 & 0.01028 & 0.06695 \\
DISC & 0.18181 & 0.25408 & 0.00009 & 0.00105 \\
DPP & -0.02086 & 0.00964 & 0.06098 & 0.12205 \\
MRFIT & 0.00043 & 0.00608 & 0.15329 & 0.13568 \\
PREMIER & -0.01004 & 0.01002 & 0.05644 & 0.12043 \\
TOHP I & -0.02998 & 0.01887 & 0.01591 & 0.08289 \\
TOHP II & -0.00820 & 0.01495 & 0.02535 & 0.09887 \\
WHI & 0.02844 & 0.00310 & 0.58964 & 0.14351 \\
\hline
\end{tabular}

Summary parameter estimate $-0.0033(S E=0.0082), p=0.6897$.

$Q$ statistic for heterogeneity with 9 degrees of freedom $=59.52, p<0.001$.

an estimated location parameter of $\Theta=162$ days (meaning $37 \%$ remain in the study, $95 \% \mathrm{CI}=114,230$ days) (21). This example demonstrates a significantly reduced survival on study compared to the large trials that we examined. This difference may be reflective of the differences in resources available between large and small studies. Therefore, our results should be interpreted with caution in terms of applicability to smaller studies. A further limitation of analysis using these types of datasets is the lack of standard ways of coding certain variables often of interest, e.g., marital status (how to analyze "married" vs. "marriage-like relationship"?) and the differing diagnostic criteria used to identify some types of comorbidities. While progress is being made in some arenas of research, more work in standardization remains (22).

Additionally, we focused our analysis on randomized trials of weight loss interventions using traditional diet and/or exercise interventions, which may have very different attrition characteristics than those of observational trials, or of RCTs of weight loss drugs or surgical interventions. Therefore, our results may not be 
Table 7 | Summary of effect estimates for female gender vs. male by study and estimates of random effects in meta-analyses of dropout.

\begin{tabular}{lcccc}
\hline Study & $\begin{array}{l}\text { Parameter } \\
\text { estimate }\end{array}$ & $\begin{array}{l}\text { Standard } \\
\text { error }\end{array}$ & $\begin{array}{l}\text { Weight in fixed } \\
\text { effects model }\end{array}$ & $\begin{array}{l}\text { Weight in random } \\
\text { effects model }\end{array}$ \\
\hline ACT & 0.52895 & 0.24405 & 0.13221 & 0.14837 \\
LRC & - & - & & \\
DASH & 0.18547 & 0.50574 & 0.03079 & 0.05190 \\
DISC & 0.41449 & 0.36164 & 0.06021 & 0.08859 \\
DPP & -0.27767 & 0.18049 & 0.21473 & 0.19932 \\
MRFIT & - & - & & \\
PREMIER & 0.41463 & 0.19094 & 0.21599 & 0.18994 \\
TOHP I & 0.35147 & 0.26206 & 0.11467 & 0.13658 \\
TOHP II & 0.18377 & 0.19628 & 0.20440 & 0.18529 \\
WHI & - & - & &
\end{tabular}

Summary parameter estimate 0.23303 (SE $=0.1251), p=0.0657$.

$Q$ statistic for heterogeneity with 6 degrees of freedom $=10.77, p=0.0955$.

Table 8 | Summary of effect estimates for black race vs. white by study and estimates of random effects in meta-analyses of dropout.

\begin{tabular}{lrrcc}
\hline Study & $\begin{array}{l}\text { Parameter } \\
\text { estimate }\end{array}$ & \multicolumn{1}{l}{$\begin{array}{l}\text { Standard } \\
\text { error }\end{array}$} & $\begin{array}{l}\text { Weight in fixed } \\
\text { effects model }\end{array}$ & $\begin{array}{l}\text { Weight in random } \\
\text { effects model }\end{array}$ \\
\hline ACT & 0.04334 & 0.26282 & 0.03518 & 0.06413 \\
LRC & -0.34757 & 0.45305 & 0.01184 & 0.02352 \\
DASH & 0.18448 & 0.55379 & 0.00792 & 0.01598 \\
DISC & 0.16821 & 0.53303 & 0.00855 & 0.01721 \\
DPP & 0.39616 & 0.18966 & 0.06756 & 0.11048 \\
MRFIT & 0.02200 & 0.13792 & 0.12777 & 0.17541 \\
PREMIER & 0.07304 & 0.17836 & 0.07640 & 0.12152 \\
TOHP I & 0.02583 & 0.32479 & 0.02304 & 0.04387 \\
TOHP II & -0.31392 & 0.26873 & 0.03365 & 0.06167 \\
WHI & 0.29874 & 0.06302 & 0.60808 & 0.36619 \\
\hline
\end{tabular}

Summary parameter estimate $0.1481(S E=0.0711), p=0.0373$.

$Q$ statistic for heterogeneity with 9 degrees of freedom $11.42 p=0.2477$

generalizable to these types of analyses due to such reasons as selfselection bias [e.g., as discussed in Ref. (23) who reported higher DORs in younger people] and differing amounts of weight lost that can be observed in drug or surgery trials [e.g., as reported in Ref. (24) who reported younger patients and those with lower BMI at greater risk for dropout of a treatment program]. Weight loss drug trials may have different factors such as side effects, or dissatisfaction with being assigned to the placebo group, causing different retention challenges. Our analysis did not include datasets from these types of trials but hopefully in the future, such data will be made publicly available.

With the presently increasing mean age of the US population, there may be interest in testing weight loss interventions in older samples and researchers should examine and plan for ways to increase study retention in older participants. Further, for studies that will include persons in the obese class II (BMI = 3539.9) and III (BMI > 40) categories, non-traditional interventions and retention strategies may need to be employed to increase

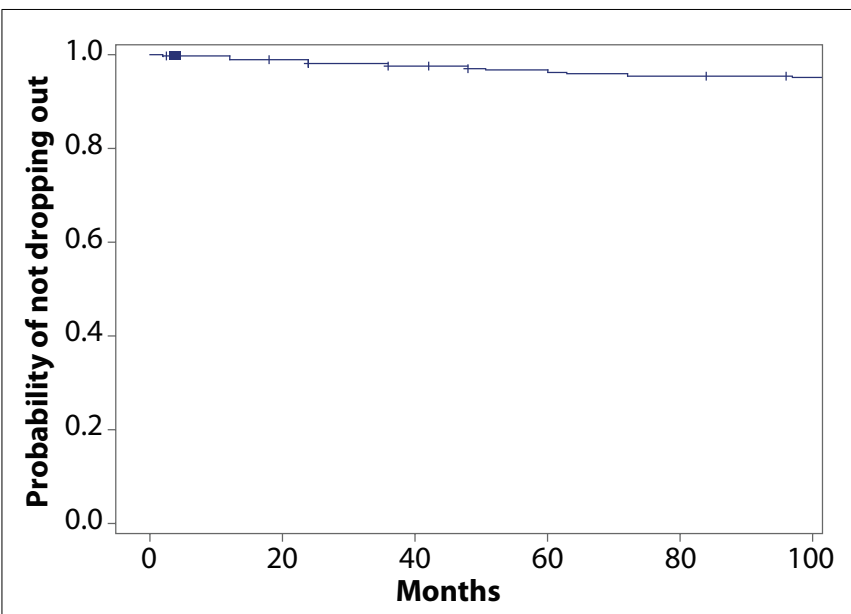

FIGURE 1 | Study survival probability (by not dropping out of the study) including all subjects from pooled analyses $(N=75,764)$.

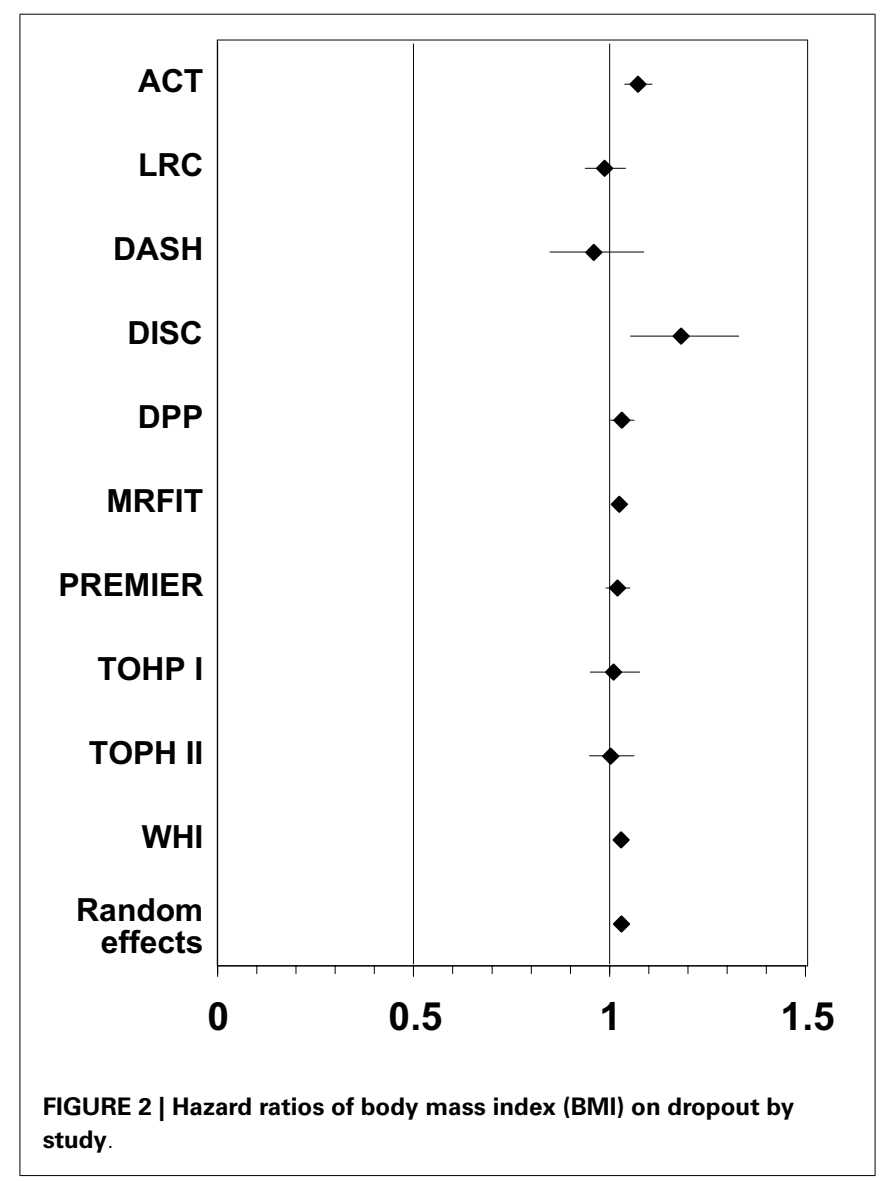

our understanding of the effectiveness of weight loss interventions similar to those we examined. The finding among these datasets that being female was associated with higher DORs cannot be explained by the data provided in the datasets used. Researchers may wish to engage in the practice of performing 

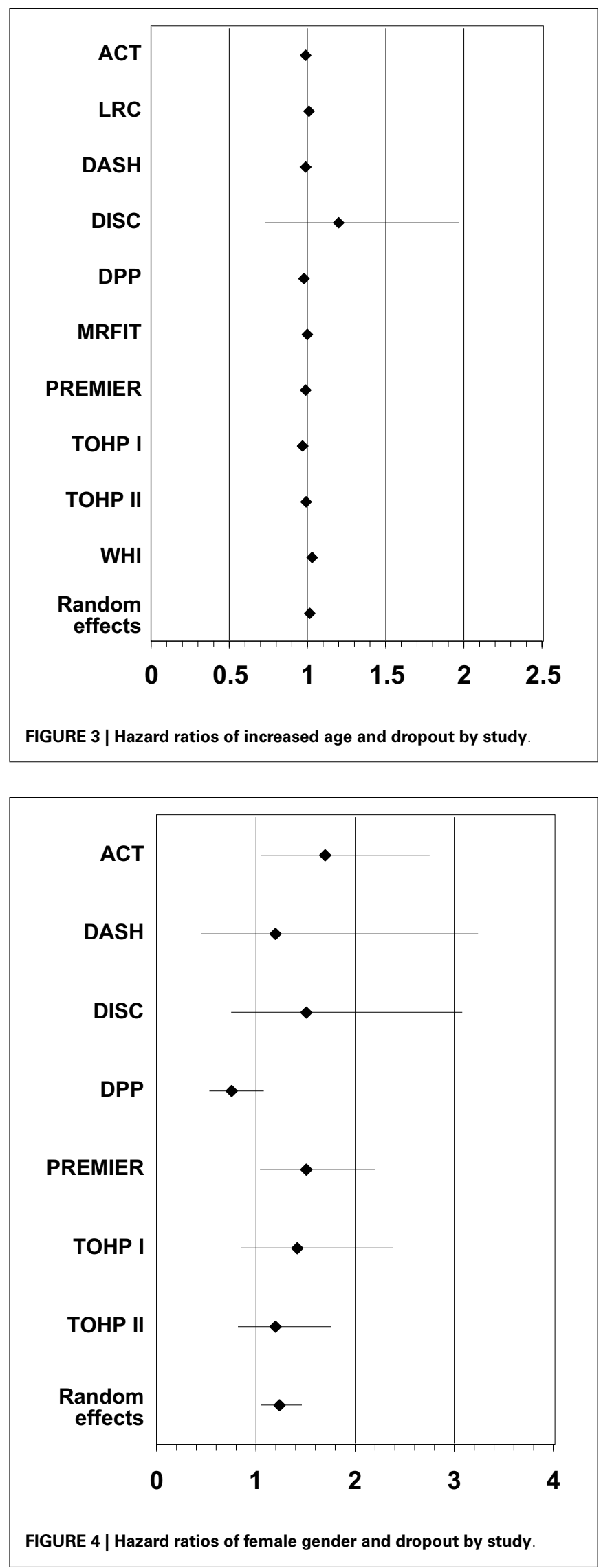

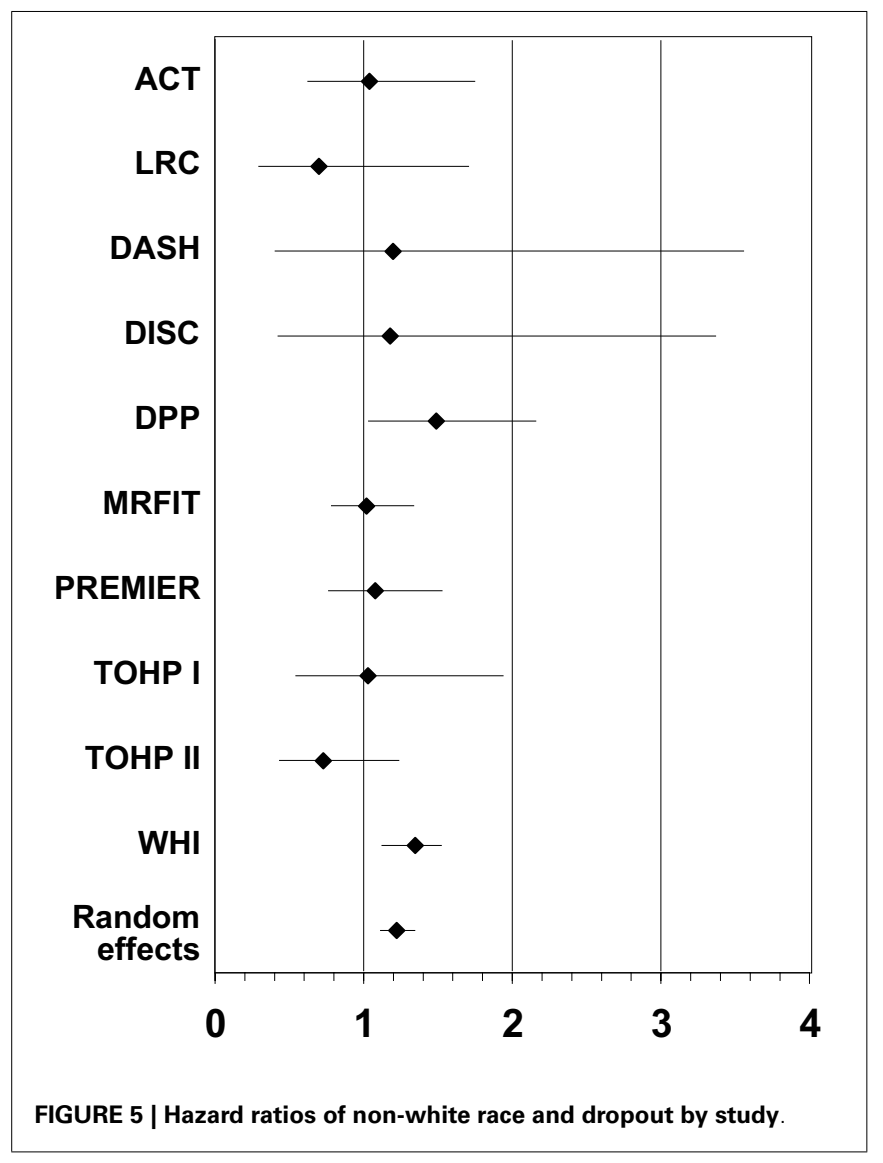

exit interviews in order to understand the true reasons participants dropped out of the study. Similarly, since there is increasing interest in understanding the racial disparities of obesity in the US, researchers would benefit from designing ways to improve retention and gather regular feedback before a participant drops out of a study so that alternatives can be collaboratively explored.

\section{ACKNOWLEDGMENTS}

This research was supported in part by National Institutes of Health (NIH) Grants R01DK078826, P30DK056336, T32HL079888, T32HL007457, and T32DK062710. The views expressed are those of the authors and not necessarily those of the NIH, or any other organization. The Diabetes Prevention Program (DPP) was conducted by the DPP Research Group and supported by the National Institute of Diabetes and Digestive and Kidney Diseases (NIDDK), the General Clinical Research Center Program, the National Institute of Child Health and Human Development (NICHD), the National Institute on Aging (NIA), the Office of Research on Women's Health, the Office of Research on Minority Health, the Centers for Disease Control and Prevention (CDC), and the American Diabetes Association. The data (and samples) from the DPP were supplied by the NIDDK Central Repositories. This manuscript was not prepared under the auspices of the DPP and does not represent analyses or conclusions of the DPP Research Group, the NIDDK Central Repositories, or the NIH. 


\section{REFERENCES}

1. Elobeid MA, Padilla MA, McVie T, Thomas O, Brock DW, Musser B, et al. Missing data in randomized clinical trials for weight loss: scope of the problem, state of the field, and performance of statistical methods. PLoS One (2009) 4:e6624. doi:10.1371/journal.pone.0006624

2. Honas JJ, Early JL, Frederickson DD, O'Brien MS. Predictors of attrition in a large clinic-based weight-loss program. Obes Res (2003) 11:888-94. doi:10.1038/oby.2003.122

3. Kong W, Langlois MF, Kamga-Ngande C, Gagnon C, Brown C, Baillargeon JP. Predictors of success to weight-loss intervention program in individuals at high risk for type 2 diabetes. Diabetes Res Clin Pract (2010) 90:147-53. doi:10.1016/j.diabres.2010.06.031

4. Mutsaerts MA, Kuchenbecker WK, Mol BW, Land JA, Hoek A. Dropout is a problem in lifestyle intervention programs for overweight and obese infertile women: a systematic review. Hum Reprod (2013) 28:979-86. doi:10.1093/humrep/det026

5. N.H.L.B.I. National Heart, Biologic Specimen and Data Repository Information Coordinating Center, Department of Health and Human Services. Available from: https://biolincc.nhlbi.nih.gov/home/

6. The effects of nonpharmacologic interventions on blood pressure of persons with high normal levels. Results of the trials of hypertension prevention, phase I. JAMA (1992) 267:1213-20. doi:10.1001/jama.1992.03480090061028

7. Efficacy and safety of lowering dietary intake of fat and cholesterol in children with elevated low-density lipoprotein cholesterol. The dietary intervention study in children (DISC). The writing group for the disc collaborative research group. JAMA (1995) 273:1429-35. doi:10.1001/jama.273.18.1429

8. Effects of weight loss and sodium reduction intervention on blood pressure and hypertension incidence in overweight people with high-normal blood pressure. The trials of hypertension prevention, phase II. The trials of hypertension prevention collaborative research group. Arch Intern Med (1997) 157:657-67.

9. Appel LJ, Champagne CM, Harsha DW, Cooper LS, Obarzanek E, Elmer PJ, et al. Writing Group of the, Effects of comprehensive lifestyle modification on blood pressure control: main results of the PREMIER clinical trial. JAMA (2003) 289:2083-93. doi:10.1001/jama.289.16.2083

10. Appel LJ, Moore TJ, Obarzanek E, Vollmer WM, Svetkey LP, Sacks FM, et al. A clinical trial of the effects of dietary patterns on blood pressure. DASH collaborative research group. N Engl J Med (1997) 336:1117-24. doi:10.1056/ NEJM199704173361601

11. Blair SN, Applegate WB, Dunn AL, Ettinger WH, Haskell WL, King AC, et al. Activity counseling trial (ACT): rationale, design, and methods. Activity counseling trial research group. Med Sci Sports Exerc (1998) 30:1097-106. doi:10.1097/00005768-199807000-00012

12. Kjelsberg MO, Cutler JA, Dolecek TA. Brief description of the multiple risk factor intervention trial. Am J Clin Nutr (1997) 65:191S-5S.

13. Knowler WC, Barrett-Connor E, Fowler SE, Hamman RF, Lachin JM, Walker EA, et al. Reduction in the incidence of type 2 diabetes with lifestyle intervention or metformin. N Engl J Med (2002) 346:393-403. doi:10.1056/NEJMoa012512

14. Rifkind BM. Lipid research clinics coronary primary prevention trial: results and implications. Am J Cardiol (1984) 54:30C-4C. doi:10.1016/0002-9149(84) 90854-3
15. Tinker LF, Bonds DE, Margolis KL, Manson JE, Howard BV, Larson J, et al. Low-fat dietary pattern and risk of treated diabetes mellitus in postmenopausal women: the Women's Health Initiative randomized controlled dietary modification trial. Arch Intern Med (2008) 168:1500-11. doi:10.1001/archinte.168.14. 1500

16. Writing group for the activity counseling trial research, Effects of physical activity counseling in primary care: the activity counseling trial: a randomized controlled trial. JAMA (2001) 286:677-87. doi:10.1001/jama.286.6.677

17. Lin DY, Wei LJ, Ying Z. Checking the Cox model with cumulative sums of martingale-based residuals. Biometrika (1993) 80:557-72. doi:10.1007/s10985008-9082-4

18. Therneau TM, Grambsch PM, Fleming TR. Martingale-based residuals for survival models. Biometrika (1990) 77:147-60. doi:10.1093/biomet/77.1.147

19. DerSimonian R, Laird N. Meta-analysis in clinical trials. Control Clin Trials (1986) 7:177-88. doi:10.1016/0197-2456(86)90046-2

20. Obesity: preventing and managing the global epidemic. Report of a WHO consultation. World Health Organ Tech Rep Ser (2000) 894:1-253.

21. Landers PS, Landers TL. Survival analysis of dropout patterns in dieting clinical trials. J Am Diet Assoc (2004) 104:1586-8. doi:10.1016/j.jada.2004.07.030

22. Clinical Data Interchange Standards Consortium. (2014). Available at: http: //www.cdisc.org/standards-and-implementations

23. Hemmingsson E, Johansson K, Eriksson J, Sundstrom J, Neovius M, Marcus C. Weight loss and dropout during a commercial weight-loss program including a very-low-calorie diet, a low-calorie diet, or restricted normal food: observational cohort study. Am J Clin Nutr (2012) 96:953-61. doi:10.3945/ajcn.112.038265

24. Gill RS, Karmali S, Hadi G, Al-Adra DP, Shi X, Birch DW. Predictors of attrition in a multidisciplinary adult weight management clinic. Can J Surg (2012) 55:239-43. doi:10.1503/cjs.035710

Conflict of Interest Statement: The Review Editor Amanda Willig declares that, despite being affiliated to the same institution as authors Kathryn Ann Kaiser, Olivia Affuso, Renee Desmond, and David B Allison, the review process was handled objectively and no conflict of interest exists. The authors declare that the research was conducted in the absence of any commercial or financial relationships that could be construed as a potential conflict of interest.

Received: 11 July 2014; accepted: 24 November 2014; published online: 18 December 2014.

Citation: Kaiser KA, Affuso O, Desmond R and Allison DB (2014) Baseline participant characteristics and risk for dropout from 10 obesity randomized controlled trials: $a$ pooled analysis of individual level data. Front. Nutr. 1:25. doi: 10.3389/fnut.2014.00025 This article was submitted to Nutrition Methodology, a section of the journal Frontiers in Nutrition.

Copyright (C) 2014 Kaiser, Affuso, Desmond and Allison. This is an open-access article distributed under the terms of the Creative Commons Attribution License (CC BY). The use, distribution or reproduction in other forums is permitted, provided the original author(s) or licensor are credited and that the original publication in this journal is cited, in accordance with accepted academic practice. No use, distribution or reproduction is permitted which does not comply with these terms. 